\title{
Computerized Data Reduction and Analysis in Positron Annihilation Coincidence Doppler Broadening Spectroscopy
}

\author{
A. Harrich, S. Jagsch, S. Riedler and W. Rosinger \\ Department of Automotive Engineering \\ FH Joanneum University of Applied Sciences \\ Alte Poststr. 149, A-8020 Graz, Austria
}

Received: October 9, 2003 Accepted: November 17, 2003

\begin{abstract}
Positron annihilation spectroscopy is a sensitive probe for studying the electronic structure of defects in solids. The high-momentum part of the Doppler-broadened spectra can be used to distinguish different elements at the annihilation site. This can be achieved by using a twodetector coincidence system, which reduces the peak to background ratio dramatically. The coincident events have to be extracted from a two-dimensional spectrum that is recorded by two high-purity germanium detectors. For this purpose the computer program MePASto was developed, which allows an automated data reduction from such Doppler-coincidence spectra, supplemented by a post-processing unit for data analysis. Additionally a case study of the identification of defect sites in an intermetallic compound is presented.
\end{abstract}

\section{INTRODUCTION}

Positron annihilation spectroscopy (PAS) is a sensitive probe for studying defects in solids. The method relies on the propensity of positrons to become localized at open-volume regions of a solid and the emission of annihilation gamma rays that escape the system without any interaction. These gamma quanta hold information about the electronic environment around the an-nihilation site [1].

The positrons are emitted from a radioactive source to a solid. The most common source is ${ }^{22} \mathrm{Na}$, for which the maximum energy of the emitted positrons is $0.54 \mathrm{MeV}$. Therefore the penetration depth is sufficient to probe the bulk. The positron slows down and thermalizes in the solid within a few picoseconds. After diffusing around the lattice, the thermalized positron annihilates with an electron creating two gamma quanta of energies of $511 \mathrm{keV}$, which is equivalent to the rest mass of the particles. The two quanta are emitted at approximately opposite directions. The deviation from $180^{\circ}$ is caused by the momentum of the electron, since the momentum of the positron is negligible due to thermalization. In the case of core electrons the momentum is large while for the valence electrons it is small.

Several observables are employed in PAS to characterize defects: positron lifetime, two-dimensional angular correlation radiation (2D-ACAR), and Doppler broadening. Positron lifetime experiments are capable of distinguishing different kinds of defects but provide no direct information on chemical variations. 2D-ACAR data allow the characterization of defects through the analysis of the momentum distribution of conduction- and valence-band electrons in the perturbing fields of defects, which requires detailed calculations of positron wave functions and the electronic band structure. Doppler broadening of annihilation radiation provides a sensitive method of defect characterization by measuring the momentum distribution of the electrons. Unlike 2D-ACAR, sensitive mainly to low-momentum electrons, it allows the examina-tion of high-momentum core electrons. The core electrons of the atoms 
near a defect site retain the properties of the free atoms, including the momentum distribution [2].

The principle of the method lies in the analysis of the positron annihilation line shape, which directly corresponds to the distribution of momentum of electronpositron pairs. The momentum itself is measured from the amount of the Doppler shift of the emitted photons. Because the positrons are thermalized, the total energy of the annihilation gamma rays is given by $2 m_{0} c^{2}-E_{\mathrm{B}}$, where $m_{0} c^{2}$ is the electron rest mass energy and $E_{\mathrm{B}}$ is the electron binding energy. When there is a net center of mass energy associated with the annihilating pair, this total energy is not split equally among the two gamma rays. One gamma ray is upshifted while the other is downshifted from the center energy of $m_{0} c^{2}-E_{\mathrm{B}} / 2$ by an amount given by $\Delta E=p_{\mathrm{L}} c / 2$, where $p_{\mathrm{L}}$ is the longitudinal component of the electronpositron momentum along the direction of the gamma ray emission. Since the direction of the gamma ray emission is random, a detector located in a given direction will record both upshifted and downshifted gamma rays. This produces an overall Doppler broadening of the annihilation peak.

In a solid, positrons predominately annihilate with outer electrons from conduction and valence bands. The analysis of the low probability events occurring from annihilation with the core electrons is not feasible in a traditional, single-detector Doppler-broadening setup due to the high level of the background. This difficulty can be overcome in a two-detector system detecting both annihilation photons and selecting only simultaneous events, thus eliminating random background counts [3].

Figure 1 shows a two-dimensional spectrum recorded from two aluminum plates of about $1 \mathrm{~cm}^{2}$ and a thickness of 1 $\mathrm{mm}$, sandwiching the positron source. For every coincident event, the energies of both gamma rays (denoted by $E_{0}$ and $E_{1}$ ) are registered in two high-purity $G e$ detectors arranged at $180^{\circ}$ to each other on both sides of the aluminum plates. These energies form the vertical and horizontal axes, and the count corresponding to each $E_{0}$ and $E_{1}$ combination is indicated in color, depending on their absolute values. The intense central peak centered at $E_{0}=E_{1}=$ $511 \mathrm{keV}$ corresponds to annihilation with

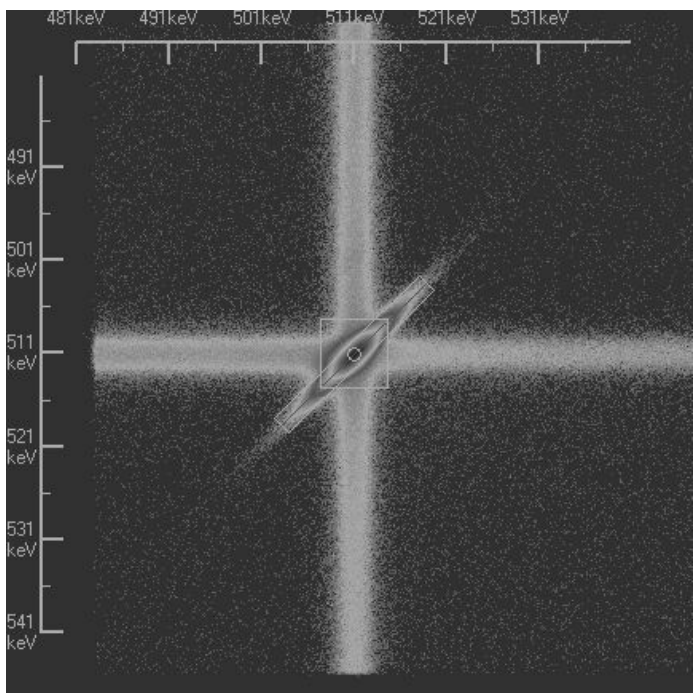

Figure 1. A two-dimensional display of coincident events collected with aluminum samples. The spectrum contains a total of $33 \times 10^{6}$ events.

valence electrons. The elliptical region extending diagonally with $E_{0}+E_{1} \approx 2 m_{0} c^{2}=$ $1022 \mathrm{keV}$ originates from annihilations with high momentum electrons, and this region is nearly background free. A cut along the diagonal can then be analyzed to observe variations in shape due to the contributions of core electrons.

Due to different detector efficiencies, the actual axis of coincidence is in general not exactly the diagonal of the twodimensional data array. The aim of this work is to provide a tool that enables the locating of this axis and the representation of the Doppler coincidence momentum distribution for further analysis.

\section{DATA REDUCTION}

The two-dimensional spectrum is available as a plain ASCII file in the form of a matrix, typically consisting of about $1000 \mathrm{x}$ 1000 data set entries. It is not necessarily quadratic, even though usually the same number of channels of the multi channel analyzer is chosen for each detector. The entries of detector "0" are displayed vertically, and the entries of detector " 1 " are represented by the horizontal axis.

The main task of the data reduction routine MePASto is the localization of the central peak, which represents the $511 \mathrm{keV}$ rest mass of the electrons, and the $E_{0}+E_{1}=$ 


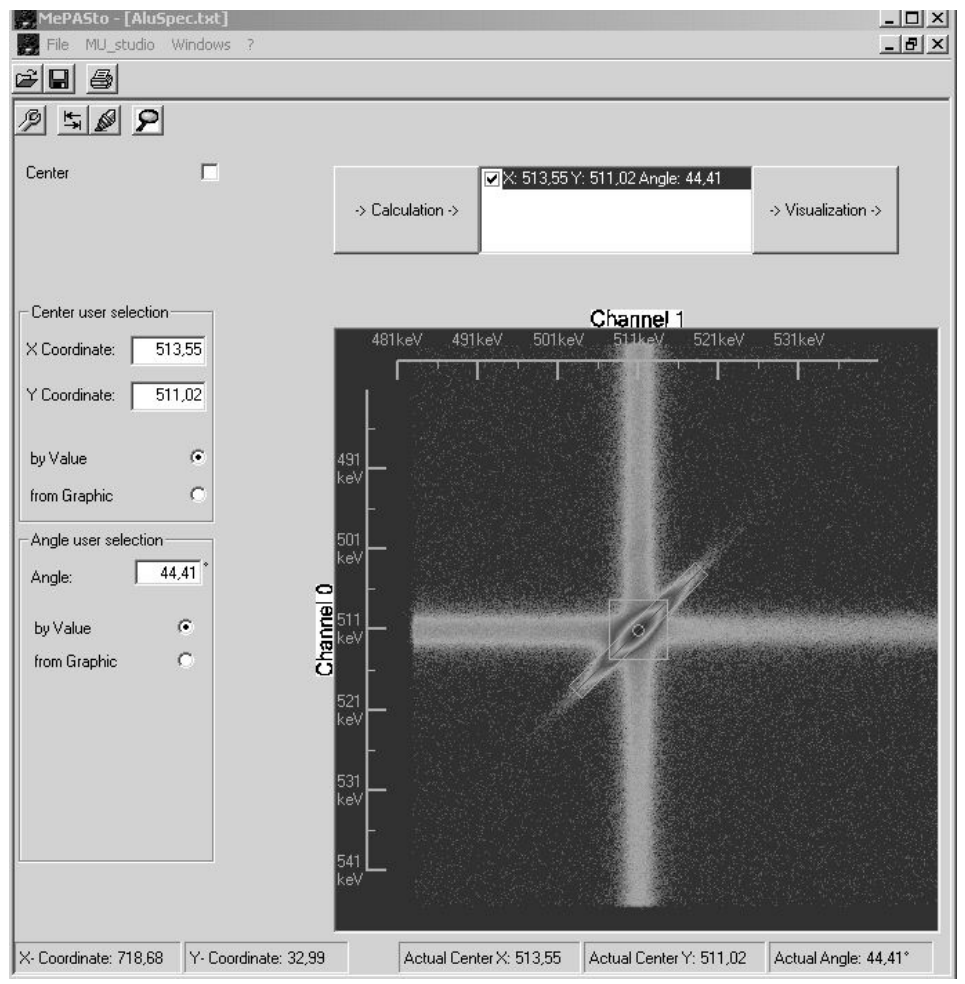

Figure 2. MePASto's graphical user interface. MS Windows standards have been applied to the icon arrangement for comfortable and easy use.

$1022 \mathrm{keV}$ axis of the coincident events. Since the data of the Doppler Coincidence Spectroscopy are represented as a digital picture, where the entries of the detectors can be regarded as pixels with $x$ and $y$ coordinates, picture-processing algorithms seem to be best suited for this task. The increment of one pixel corresponds to the energy difference of two channels of the multi-channel analyzer. The pixel to energy scaling is accomplished by taking the difference of the channel number, representing the $511 \mathrm{keV}$ peak, and the zero energy channel.

The data processing starts with the search for ten maximum entries of the data area in order to find the region of the center of the spectra. For this task a fast sorting algorithm is indicated. With the coordinates of these pixels a first approach for the center can then be achieved by simply averaging. As a next step a predefined area of $100 \mathrm{x}$ 100 pixels encompassing this maximum entry is chosen to calculate the center of gravity, which is then assigned to the actual $511 \mathrm{keV}$ peak (the square and open circle at the center of Figure 1). Additionally the program enables the user to determine the peak by simply mouse clicking the 2D spectrum, which is done most promisingly within linear scaling of the picture.

The coincident region of the Doppler broadening spectra corresponds to a projection onto the $E_{0}+E_{1}\left(=2 \Delta E=p_{\mathrm{L}} C\right)$ axis from Figure 1. For the search of this axis, polar coordinates centered at the 511 $\mathrm{keV}$ peak are employed. Within the angular range $40^{\circ}$ to $50^{\circ}$ the interpolated data entries are summed up along a straight line within an interval of 100 pixels in steps of one hundredth of a degree. If a pixel is only touched, an interpolation algorithm ensures that it gets the correct weight. The angle that gives the highest sum is used as the origin for a succeeding procedure. The line with the maximum sum is taken as a starting point for a least squares data fit. It is divided into intervals of adjustable width and then the maximum in a slice perpendicular to the line is located for each interval. The data points gained in this way are fitted to a straight line, which eventually represents the optimized cut in the 2D spectrum. The data fit can be performed optionally by keeping 
the $511 \mathrm{keV}$ point fixed or free as a fitting parameter. Relativistic effects on the core electrons of heavy elements result in a small deviation of the coincident events from a straight-line, which should lead to a small shift of the regression line, if the center of gravity is allowed to move.

The program also enables a userdefined cut of the $E_{0}+E_{1}$ - axis by mouse clicking in the 2D spectrum, which gives an additional degree of freedom. Parameter studies showed that the human eye is an excellent analyzing instrument. Most of the visually determined cuts along the coincidence axis resulted in spectra with no significant deflection from the calculated ones.

Different interpolation algorithms are implemented in the program. By default a bilinear interpolation algorithm is in use, where only the nearest neighbor pixel

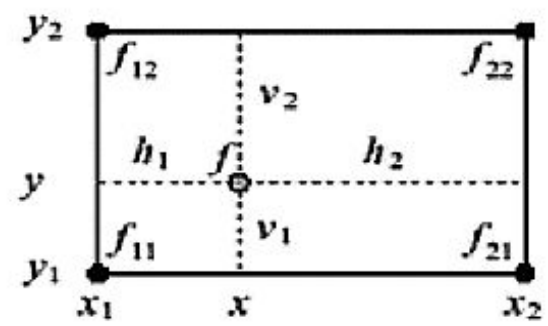

Figure 3. In the bilinear interpolation algorithm only the nearest neighbor pixel entries are taken into account.

entries have to be taken into account (Figure $3)$.

The interpolated function value $f(x, y)$ can be evaluated by

$$
f(x, y)=\frac{h_{1} f\left(x_{2}, y\right)+h_{2} f\left(x_{1} y\right)}{h_{1}+h_{2}}=\frac{h_{1} v_{1} f_{22}+h_{1} v_{2} f_{21}+h_{2} v_{2} f_{12}+h_{2} v_{2} f_{11}}{\left(h_{1}+h_{2}\right)\left(v_{1}+v_{2}\right)}
$$

with

$$
f\left(x_{1}, y\right)=\frac{v_{1} f\left(x_{1}, y_{2}\right)+v_{2} f\left(x_{1}, y_{1}\right)}{v_{1}+v_{2}} \quad, \quad f\left(x_{2}, y\right)=\frac{v_{1} f\left(x_{2}, y_{2}\right)+v_{2} f\left(x_{2}, y_{1}\right)}{v_{1}+v_{2}}
$$

and $f_{i j}=f\left(x_{i}, y_{j}\right)$.

A bi-cubic interpolation algorithm, which requires the specification of not only the neighboring grid points but also their derivatives [5], and an interpolation scheme, which takes the inverse distances of the neighbor pixels as weights, are additionally made available in the program.

The numerically elaborate algorithms are programmed as ANSI C dynamic link libraries (DLLs), the user interface is written in Visual Basic. Calling C routines from Visual Basic provides a convenient way to quickly develop graphical user interfaces and packages that use mathematical routines.

\section{DATA ANALYSIS}

In Figure 4 the coincidence spectrum of the aluminum measurement is shown. It corresponds to a projection onto $E_{0}-E_{1}$ (= $\left.2 \Delta E=p_{\mathrm{L}} C\right)$ axis from Fig. 1. The projection includes events in a window along the diagonal with $2 m_{0} c^{2}-0.44 \mathrm{keV}<\mathrm{E} 0+\mathrm{E} 1<$ $2 m_{0} c^{2}+0.44 \mathrm{keV}$. The width of the sum energy window is selected to exclude vertical and horizontal bands depicted in Figure 1 . The variation of the sum-energy window to $2 m_{0} c^{2} \pm 1.74 \mathrm{keV}$ did not change the shape of the annihilation spectrum, apart from an increase in the background. Up to four spectra or three spectra and one numerically simulated positron annihilation probability density [4] can be displayed simultaneously. Additionally the data analysis tool MU_studio enables the numerical integration of the spectra within arbitrary intervals (indicated by the two vertical lines in Fig. 4) for the determination of line shape parameter. 


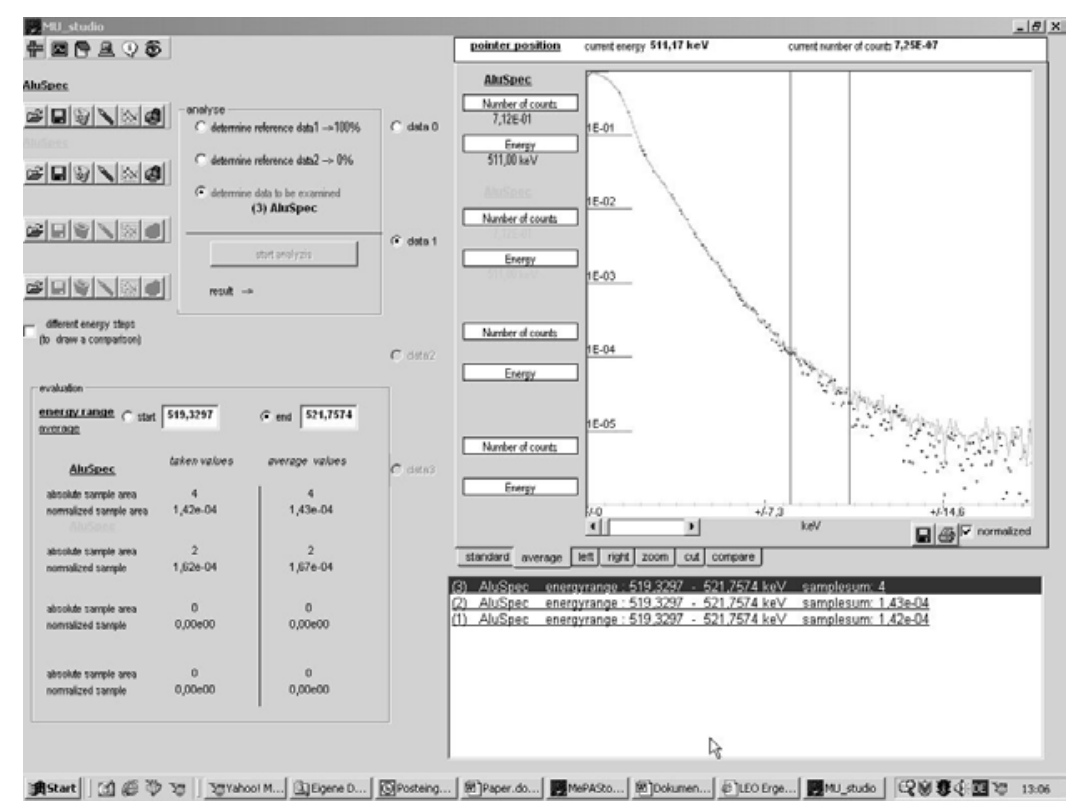

Figure 4. MePASto's data analysis tool MU_studio. In the data window the average of the 'left' and the 'right' halves of the $A$ l coincidence spectrum displayed in Figure 1 is illustrated. Up to four spectra can be displayed and analyzed at once.

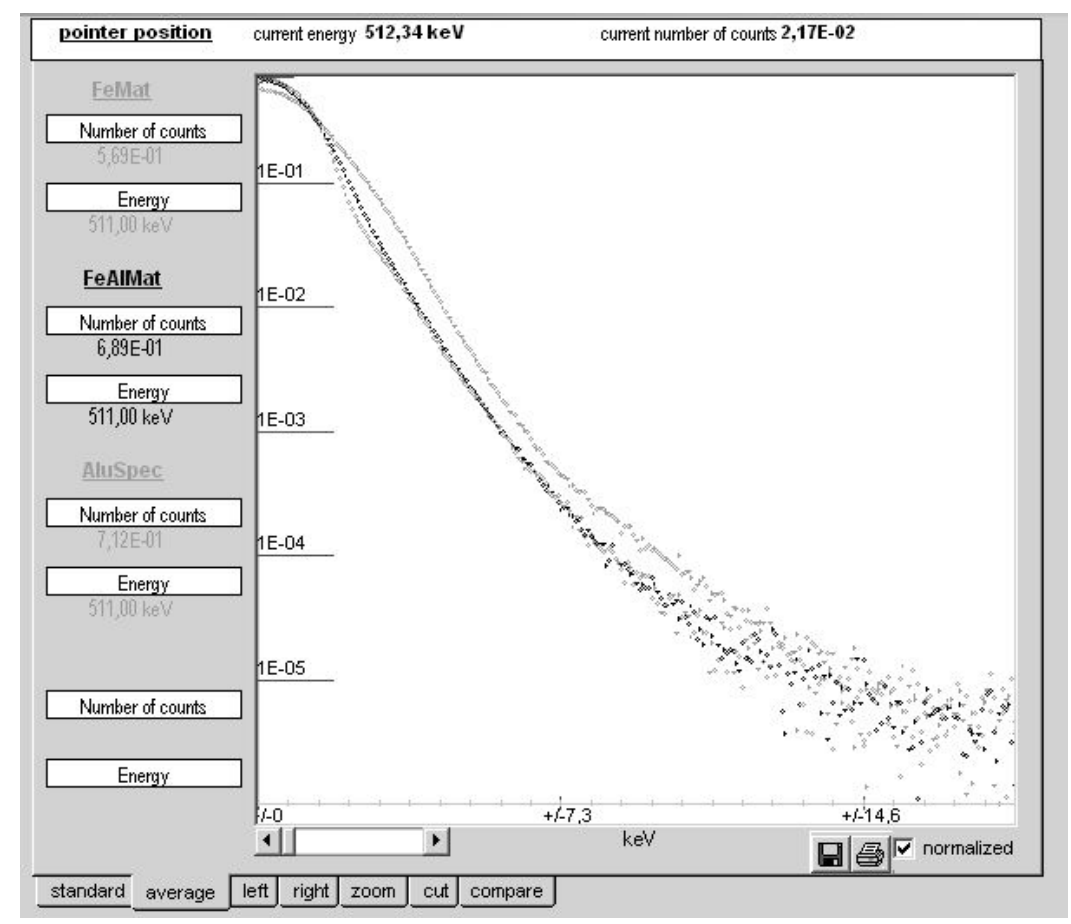

Figure 5. The comparison of the normalized spectra of pure $F e$ (upper gray curve), pure $A$ l (lower gray curve) and of $\mathrm{FeAl}$ (black curve almost covering the $A l$ spectrum) leads to the conclusion that the positron annihilation takes place at lattice sites surrounded only by $A /$ atoms. This leads to the conclusion that vacancies are predominantly located on the Fe sublattice. 
The momentum axis can be scaled either in keV or, as customary in 2D-ACAR investigations, in $\mathrm{mrad}\left(10^{-3} \mathrm{~m}_{0} \mathrm{c}\right)$. For the comparison of different spectra and of experimentally and theoretically obtained annihilation probability densities, the area under the experimental data is normalized to unity.

\section{CASE STUDY}

$\mathrm{FeAl}$ is an intermetallic compound with B2 structure, i.e. composed of two sublattices being occupied completely by $\mathrm{Fe}$ or $A /$ atoms, respectively. A vacancy on one sublattice is surrounded entirely by atoms of the second sublattice. Consequently, if positrons are trapped, e.g. by a vacancy on the $F e$ sublattice, their annihilation spectrum comprises a larger proportion of annihilation processes with core electrons of the surrounding $A l$ atoms while trapping by vacancies on the $A l$ sublattice would result in a dominating contribution of core electrons belonging to $\mathrm{Fe}$ atoms.

As in other B2 compounds of metals belonging to Group VIII and Group IIIA the concentration of thermal vacancies in $\mathrm{FeAl}$ is generally high on the transition metal sublattice. Therefore, this system is well suited to demonstrate that measurements of the high-momentum range of Doppler spectra can identify particular lattice sites. Coincidence Doppler broadening spectra have been recorded for pure $\mathrm{Al}$ and pure $\mathrm{Fe}$ as well as $\mathrm{FeAl}$ [6]. A comparison of the momentum distributions confirms the conclusion that the thermal vacancies are predominantly situated on the iron sublattice.

\section{ACKNOWLEDGEMENTS}

The authors would like to thank their supervisors G. Bischof, E. Bratschitsch and A. Casey from FH Joanneum and G. Krexner from University of Vienna for their help and encouragement. MePASto is available free of charge and can be downloaded from http://fahrzeugtechnik.fhjoanneum.at/links/MePASto.

\section{REFERENCES}

1. M. J. Puska and R. M. Nieminen, Theory of Positrons in Solids and on
Solid Surfaces, Reviews of Modern Physics 66, 841 (1994).

2. S. Szpala, P. Asoka-Kumar, B. Nielsen, J. P. Peng, S. Hayakawa, K. G. Lynn and H.-J. Gossmann, Defect Identification using core-electron contribution in Doppler-broadening spectroscopy of positron-annihilation radiation, Phys. Rev. B 54, 4722 (1996)

3. P. Asoka-Kumar, M. Alatalo, V. J. Ghosh, A. C. Kruseman, B. Nielsen and K. G. Lynn, Increased Elemental Specificity of Positron Annihilation Spectra, Phys. Rev. Lett. 772097 (1996).

4. M. Alatalo, B. Barbiellini, M. Hakala, H. Kauppinen, T. Korhonen, M. J. Puska, K. Saarinen, P. Hautojärvi and R. M. Nieminen, Theoretical and experimental study of positron annihilation with core electrons in solids, Phys. Rev. B 54, 2397 (1996).

5. W. H. Press, S. A. Teukolsky, W. T. Vetterling, and B. P.Flannery, Numerical Recipes in C - The Art of Scientific Computing, Cambridge University Press (1988).

6. W. Egger, G. Bischof, V. Gröger and G. Krexner, Identification of Defect Sites in FeAl by Doppler Spectroscopy of Core Electrons, Materials Science Forum 363-365, 82 (2001). 